\title{
The Fine Plane Range Estimation From Point Cloud
}

\author{
Natalija Chmelarova, Pavel Chmelar, Lubos Rejfek \\ Department of Electrical Engineering \\ Faculty of Electrical Engineering and Informatics, University of Pardubice \\ Pardubice, Czech Republic \\ natalija.chmelarova@student.upce.cz
}

\begin{abstract}
This paper deals with the fine plane estimation. For a plane detection the level connected component labeling algorithm is used. The precise plane position is determined by the plane's points histogram in the scanning dimension. Up till now, the final plane's range has been selected manually by a parameter. Plane presence in an input point cloud represents the higher points' concentration in the scanning dimension, higher than average value. The concentration decreases on both sides from a local maximum value. This paper presents the algorithm of the elbows' position estimation for the optimal plane's points range expression. The experimental part shows practical results of several examples and gives recommendations for parameters setting.
\end{abstract}

Keywords—plane detection, elbow estimation, level connected component labeling

\section{INTRODUCTION}

Measured points in a cloud have no relation between each other except for the common coordinate space origin. To get more information about the scanned space the additional processing is necessary. Nowadays measurement systems, as Faro Focus 3D, provide detailed and precise point clouds with big amount of measurement points, see [1]. The high memory consumption is not suitable for all systems. For example, mobile robots are usually composed from small embedded devices. If they use a 3D map for the navigation, this map has to be simplified to meet robots' memory space [2]. Likewise, when measurement data are processed, usually individual points losing their meaning. More important is an object or a structure composed from these points. The robot in this research provides the furniture contours extraction and recognition. In our research we focus on the detection of planes.

The most recent research in [3] deals with the robust road extraction by using the fusion of the LiDAR point cloud, camera images, and the deep learning technique. The presented results show the algorithm abilities for robust detection under unfavorable conditions. Authors in [4] propose new method for plane detection by use of octree-balanced density down-sampling and iterative adaptive plane extraction. Presented results show the developed method robustness. The randomized Hough transform is fundamental for plane detection in the paper [5]. The developed algorithm stands out by its speed and accuracy. One of the recent research [6] presents a complex system for the plane detection and classification. Planes are segmented by the DBSCAN method which respects also curvatures to provide the fine segmentation. The research in [7] focuses on the LiDAR camera calibration by extraction of plane markers from 3D point cloud data. By use of plane it is easier to select area then from the vertex.

In our research [8], [9], and [10] we use own modified connected component labeling algorithm for 3D space - LCCL
(Level Connected Component Labeling). A plane presence is determined by the higher points' concentration in the scanning dimension. Up till now, the final plane range has been selected manually by a parameter. In this paper we propose the method for the fine plane range estimation based on the elbows detection in the histogram. This method is known many years, see [11]. In the recent research the authors modified the method's resilience to long tails [12]. In this paper we present our adaptation for the fine plane range estimation, which uses two stages. At the first step the elbow's position is roughly estimated and at the second step, the fine searching is performed.

The rest of the paper is organized as follows. Section II briefly describes the plane detection algorithm. Section III introduces the proposed algorithm. Section VI discuss the results. The paper ends with a conclusion.

\section{Plane Detection Algorithm}

The plane detection algorithm is introduced in [8]. For the purpose of this paper it is necessary to explain how a plane is detected. The algorithm LCCL is based on the progressive searching of individual levels in the selected scanning dimension $\operatorname{dim}_{s}$ and their marking by indexes in the similar way as the classical component labeling. Fig. 1 shows one level estimation for a simple point cloud model (blue points).

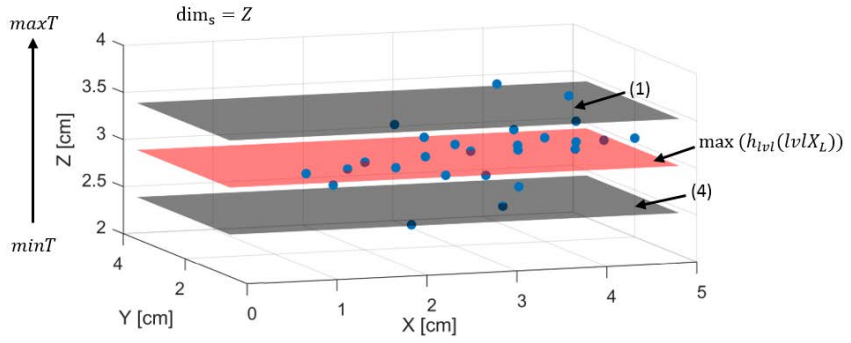

Fig. 1. Detail of plane estimation in point cloud.

Analyzed data are marked as the input matrix $X_{L}$. The process begins by setting the minimal minT and the maximal maxT input data range in the scanning dimension $\operatorname{dim}_{s}$. There are two important parameters, the $l v l S$ as the maximal deviation from a level value, and the lvlRS multiplier denoting the new level searching range. The algorithm uses two values to express the range of selected data in each step, the down threshold downT (4), and the up threshold upT (1). For the first step the downT value is set as the minimal value of the scanning dimension downT $=\min \left(\forall X_{L}\left(\operatorname{dim}_{S}\right)\right)$. The value $u p T$ is in each step set by the equation

$$
u p T=d o w n T+l v l S \cdot l v l R S .
$$

The selected point cloud data range is described in the next equation by mentioned parameters as 


$$
\begin{aligned}
\operatorname{lvl} X_{L}= & \left(\forall X_{L}\left(\operatorname{dim}_{s}\right) \geq \operatorname{downT}\right) \wedge \\
& \wedge\left(\forall X_{L}\left(\operatorname{dim}_{s}\right) \leq u p T\right) .
\end{aligned}
$$

The algorithm then estimates the level value $d L$ (red plane in Fig. 1) from the level histogram $h_{l v l}$ as $\max \left(h_{l v l}\left(l v l X_{L}\right)\right)$. The histogram of points' $Z$ dimension from Fig. 1 is shown in Fig. 2.

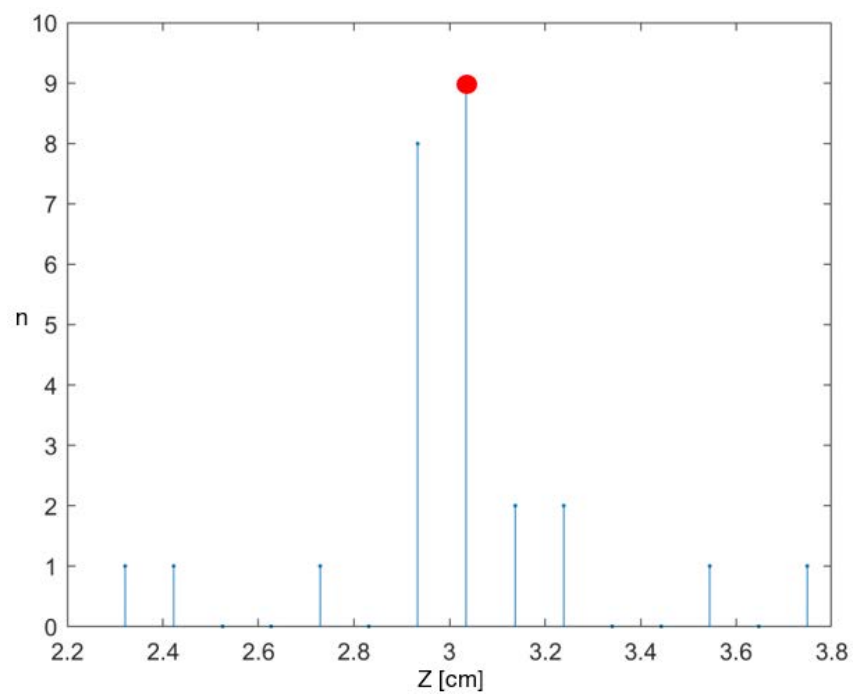

Fig. 2. Points' histogram in scanning dimension $\operatorname{dim}_{s}=Z$.

The level histogram is composed from the upT and the downT points' range (grey planes in Fig. 1) in scanning dimension $\operatorname{dim}_{s}=Z$. The rough level data range $X_{L} l v l R$ is selected by using the $l v l S$ value and the estimated level value $d L$ in the interval [downT, upT] as

$$
\begin{aligned}
X_{L} l v l R= & \forall X_{L}\left(\operatorname{dim}_{S}\right) \geq(d L-l v l S) \wedge \\
& \wedge \forall X_{L}\left(\operatorname{dim}_{S}\right) \leq(d L+l v l S) .
\end{aligned}
$$

After a level estimation at the $n$-th step the new minimum downT for the next step $(n+1)$ is defined as

$$
d o w n T=d L+l v l S,
$$

and the value $u p T$ is again set according to the equation (1). Combination of equations (1), (3) and (4) ensures best levels' distributions in the input data $X_{L}$ according to their concentrations in analyzed dimension $\operatorname{dim}_{s}$.

For plane visualization we use own developed tool the level image, introduced in [9]. The detected plane from Fig. 1 with marked plane outliners is shown in Fig. 3.

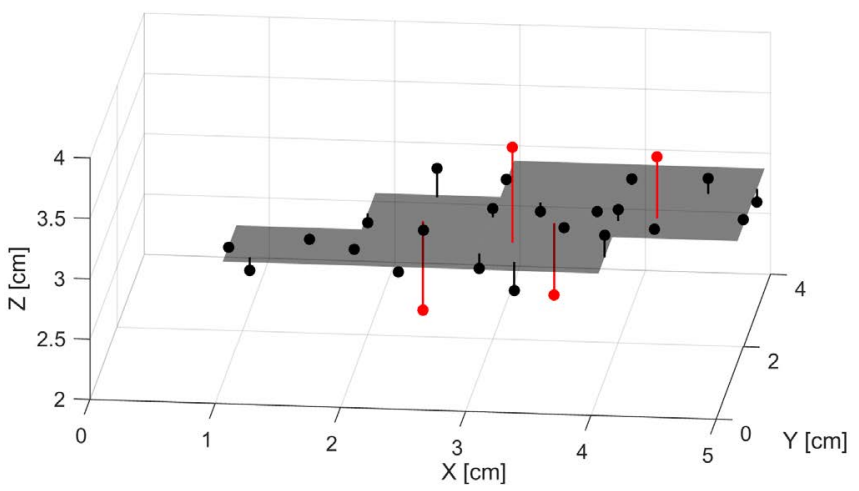

Fig. 3. Plane visualization by level image with marked outliers by red color.
For more information about the level image, its utilisation to get important plane's space properties or how to isolate individual planes in one level, see [8] and [10].

\section{Fine Plane Estimation}

The parameter $l v l S$ in equation (3) is selected by the user. It is useful to determine this parameter manually, it allows to express the maximal points' deviation from the detection level, which represents quality of the detected plane, but the selected range is not always ideal. In this section we describe the algorithm for the automatic fine plane's data range selection on the real point cloud data. The input point cloud is shown in Fig. 4. The histogram of the scanned dimension $Z$ gives Fig. 5. By its closer analysis is noticeable that there are two local maxima on both sides representing the room's floor and the ceiling. The higher concentration in the middle is caused by the higher measurement points density and by planes near to window. From a local maxima value the point's concentration decreasing on both sides to its average value in the $Z$ dimension. The histogram in Fig. 5 is noisy and before further processing it is necessary to apply filtration.

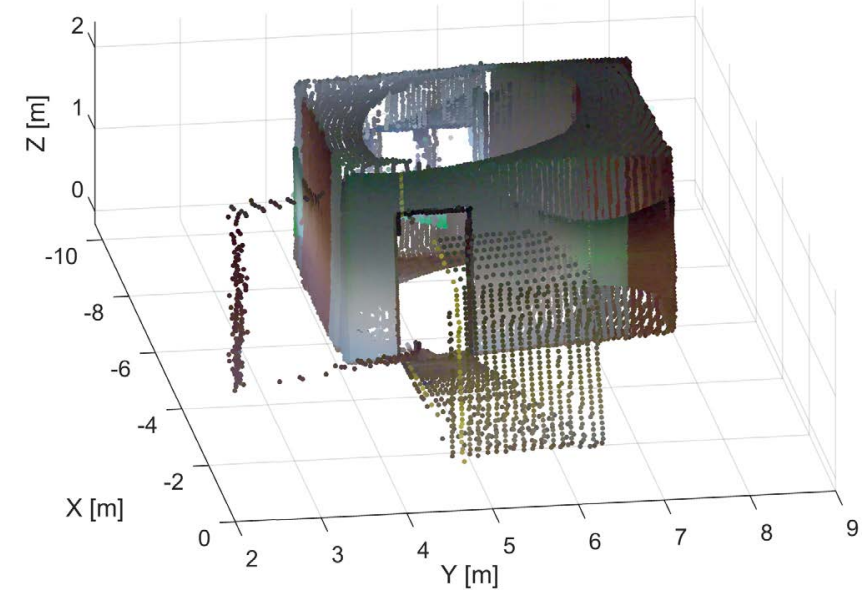

Fig. 4. Input point cloud.

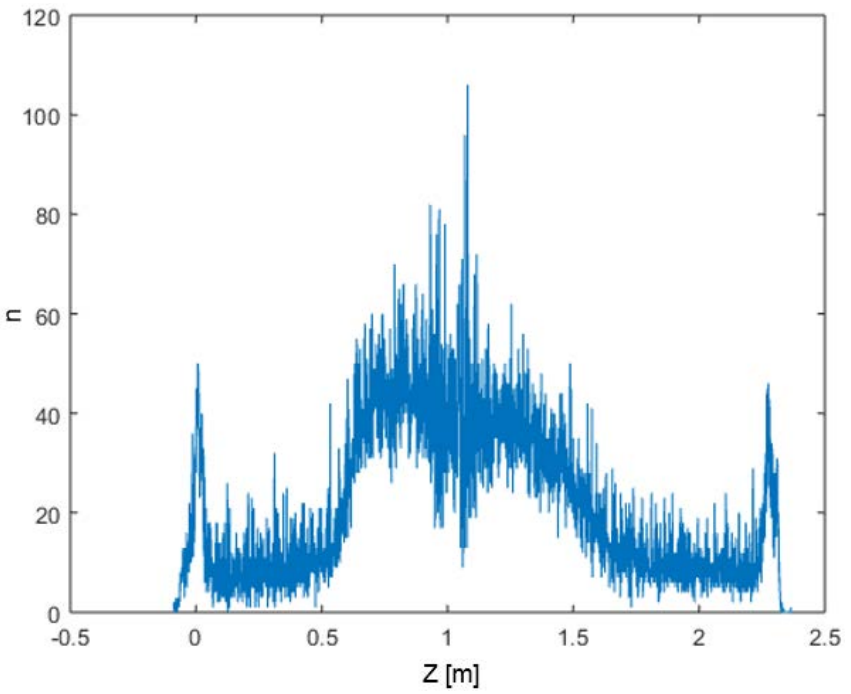

Fig. 5. Histogram of points' concentration from Fig. 4 in $Z$ axis.

Following Fig. 6 shows the histogram's detail for the first level (left side of Fig. 5) estimation, selected by the equation (3). The parameters $l v l S=0.1$ and $l v l R S=1.5$. 


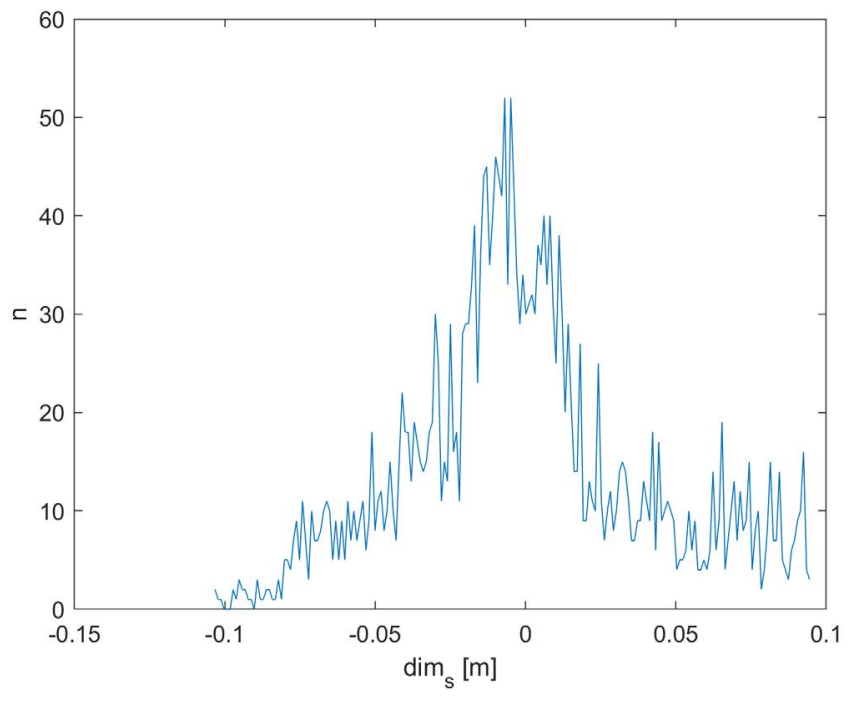

Fig. 6. Histogram of first level estimation.

The aim of the fine range selection is to find the transition from the higher concentration to the average value. The condition for start the search of elbows' position is defined by following condition

$$
\frac{\overline{h_{l v l}}}{\max \left(h_{l v l}\right)} \leq r_{l v l}
$$

where $r_{l v l}$ is the ratio between the histogram mean $\overline{h_{l v l}}$ and its maximal value $\max \left(h_{l v l}\right)$. When this ratio is higher then selected threshold $r_{l v l}$ the elbows' position is estimated. For our purpose we select $r_{l v l}=45 \%$.

The blue curve in Fig. 6 is the point density histogram near to the detected $d L$ with $1 \mathrm{~mm}$ resolution in $\operatorname{dim}_{s}$. The curve is noisy and for the filtration and further processing we use the derivation step $d s$. The green line in Fig. 7 is the moving average $m A h_{l v l}$ of the histogram $h_{l v l}$ with the window size $2 d s$. The parameter $d s=1 \mathrm{~cm}$ in this example. From the filtered curve it is possible to securely estimate positions of both elbows. The maximal value is marked by index $\max _{i d x}$.

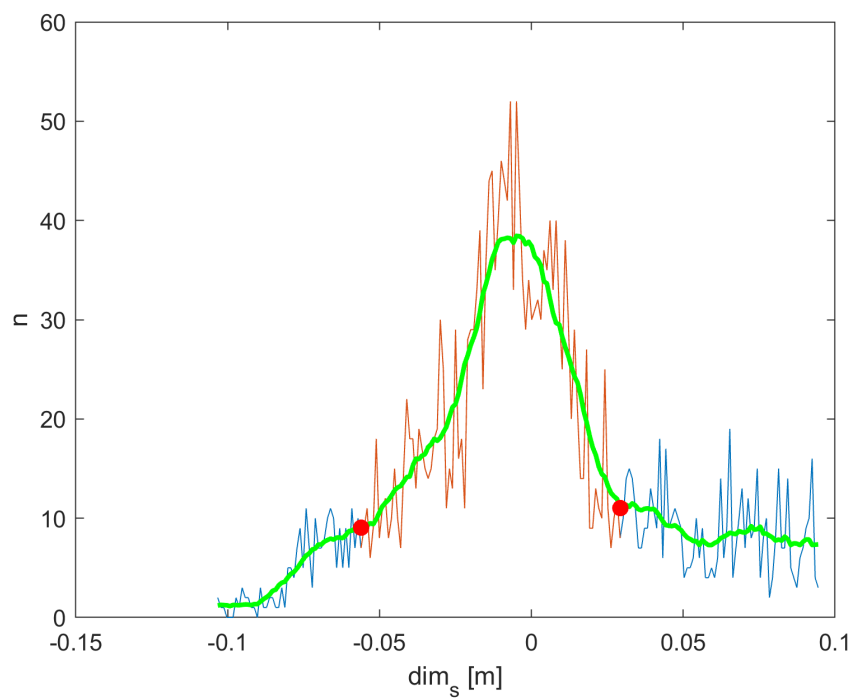

Fig. 7. Fine plane range estimation from filterd histogram. (blue curve - orginal histogram, green line - filtered histogram, red dots are new estimated range)
The fine plane estimation lies in the automatic finding the elbows' position. The first algorithm's rough step is the check of derivation $\Delta h_{l v l} / \Delta d s$ value. The derivation is evaluated in the moving window of size $d s$ from the maximal histogram value $\max \left(h_{l v l}\right)$ on both sides. The derivation is defined as follows

$$
\begin{gathered}
\frac{m A h_{l v l}\left(\max _{i d x} \pm d s \cdot n L\right)-}{\Delta d s}=\frac{-m A h_{l v l}\left(\max _{i d x} \pm d s \cdot(n L+1)\right)}{d s}
\end{gathered}
$$

where the sign \pm marks the both sides' check. The range of the index $n L$ for the right side is defined as $n L \in\left[1,2, \ldots, \max _{i d x}+n \cdot d s \leq N h_{l v l}\right]$ and for the left side as $n L \in\left[1,2, \ldots, \max _{i d x}+n . d s \geq 1\right]$. The value $N h_{l v l}$ is size of histogram elements and the index $n$ increasing from $[0,1, \ldots]$ until the condition in $n L$ is valid. The derivation value is also check by the next equation

$$
\frac{\Delta h_{l v l}}{\Delta d s}<0.25
$$

which describes the decrease of derivation value under $25 \%$.

After finding the position by equation (7) it follows the second step - the final accurate position estimation. From this position the algorithm uses the fine point by point evaluation. This step uses the index $m L$ and starts in the last position $d s \cdot n L$. The values of histogram are compared and checked by the ratio $v_{\text {diff }}$, set by the user. The following equation (8) gives the ratio calculation

$$
\frac{m A h_{l v l}\left(\max _{i d x} \pm d s \cdot n L+0,5 d s+m L\right)}{m A h_{l v l}\left(\max _{i d x} \pm d s \cdot n L+m L\right)} \leq v_{d i f f}
$$

We used $v_{\text {diff }}=5 \%$ in our experiment, which express small value change. The value of half derivation step $0,5 d s$ ensures the appropriate offset between compared values with the value on the index $m L \in[0,1, \ldots, d s]$. In case of any value does not meet the condition (8), the algorithm ends the search and the final elbow's position is on the index $m A h_{l v l}\left(\max _{i d x} \pm d s \cdot(n L+1)\right)$. Two red dots in Fig. 7 mark the elbows' position. The estimated positions are about the points' concentration equal to 10 . The detected plane before and after fine estimation is depicted in Fig. 8.
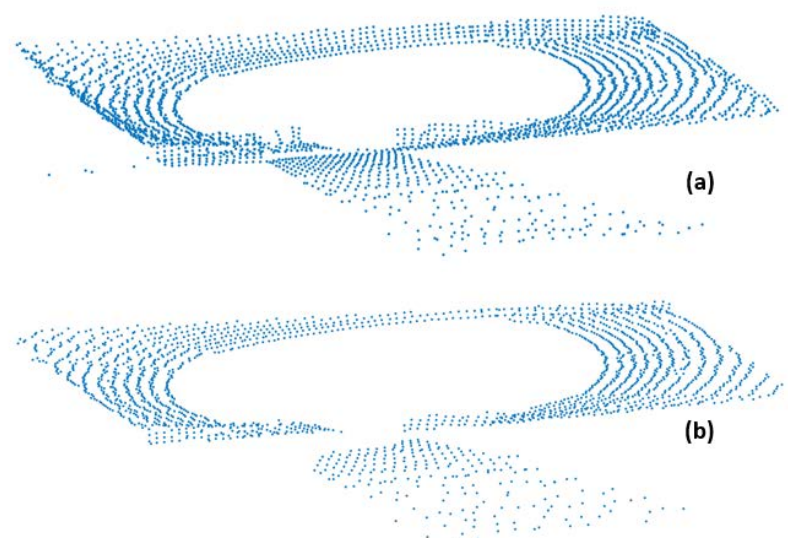

Fig. 8. Plane data renge, (a) before fine estimation, (b) after fine estimation.

From analysis of Fig. 8 (a) is obvious that a part of points belongs to planes in the $X$ and the $Y$ axis. After their removal 
in Fig. 8 (b), all present points belongs entirely to the detected plane in $Z$ axis. The decision which point belongs to the concrete plane depends on the plane's statistical parameter, for witch plane that point has better statistical properties.

\section{EXPERIMENTAL RESULTS}

This section includes several examples and situation which can happen when the new proposed method is used. The first example in Fig. 9 is indoor corridor. Plane detection parameters has bigger values than is usual, concretely $l v l S=0.3$ and $l v l R S=1.5$.

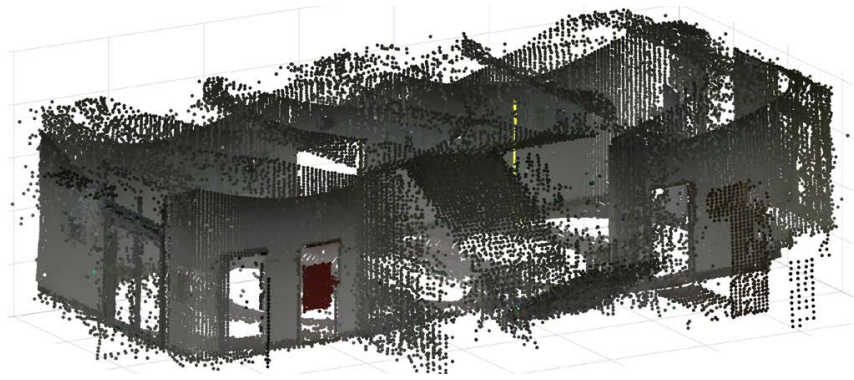

Fig. 9. Point cloud of indoor coridor

This point cloud is scanned in $Z$ dimension. The histogram of the first level with the estimated elbows marked by red dots is shown in Fig. 10.

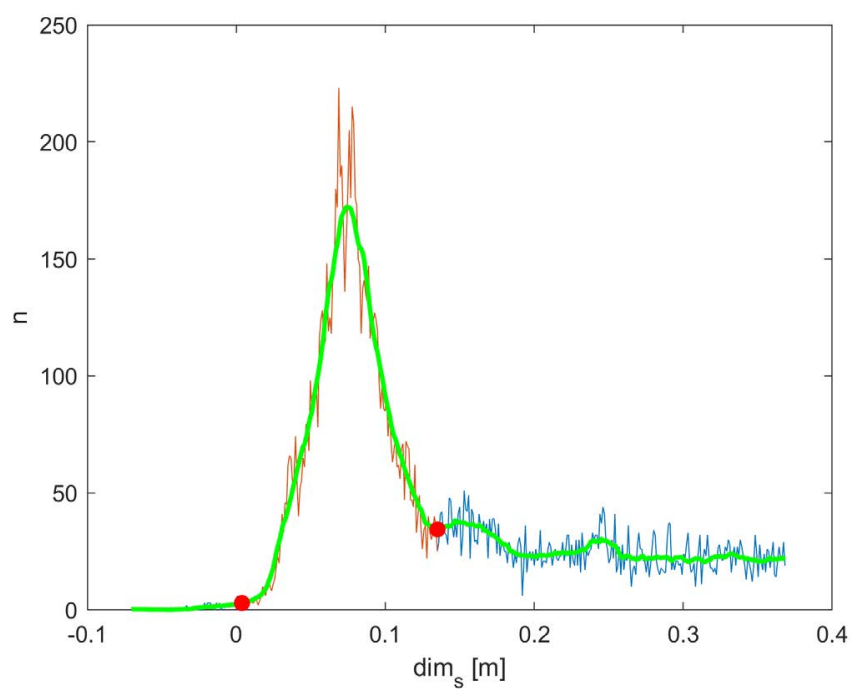

Fig. 10. First level historgram of Fig. 9 with marked elbows.

By comparing of both ranges in Fig. 11 and Fig. 12 is obvious the correct algorithms' output. Even a bigger range was selected via the parameter $l v l S$ the fine plane's range was estimated.

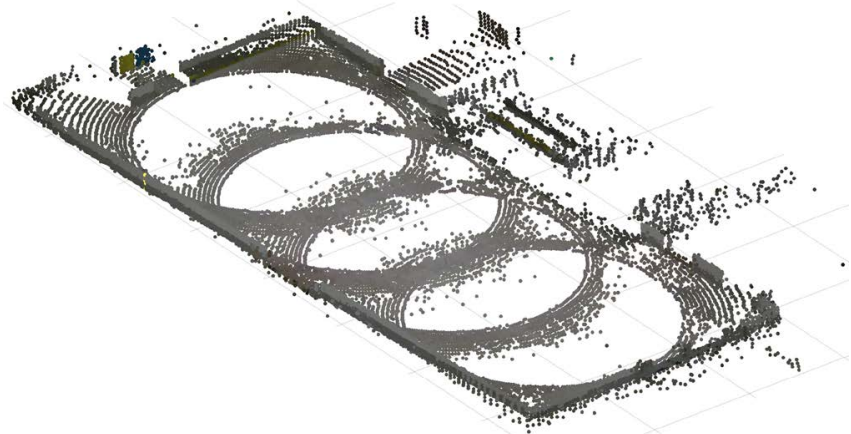

Fig. 11. First level estimation of Fig. 9.

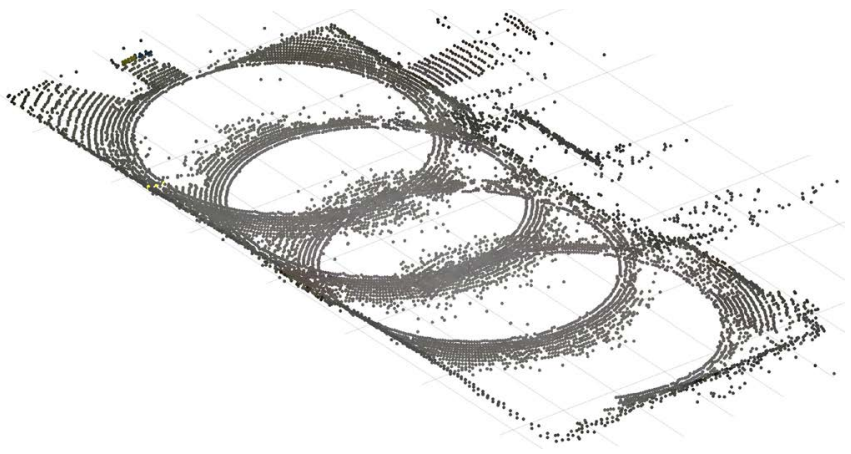

Fig. 12. Fine level estimation of Fig. 11.
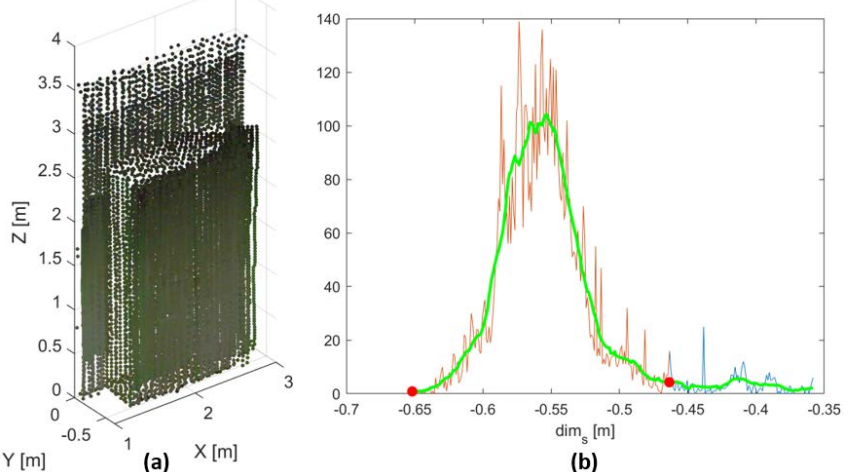

Fig. 13. Doors, (a) point cloud, (b) Y axis histogram with mared fine range.
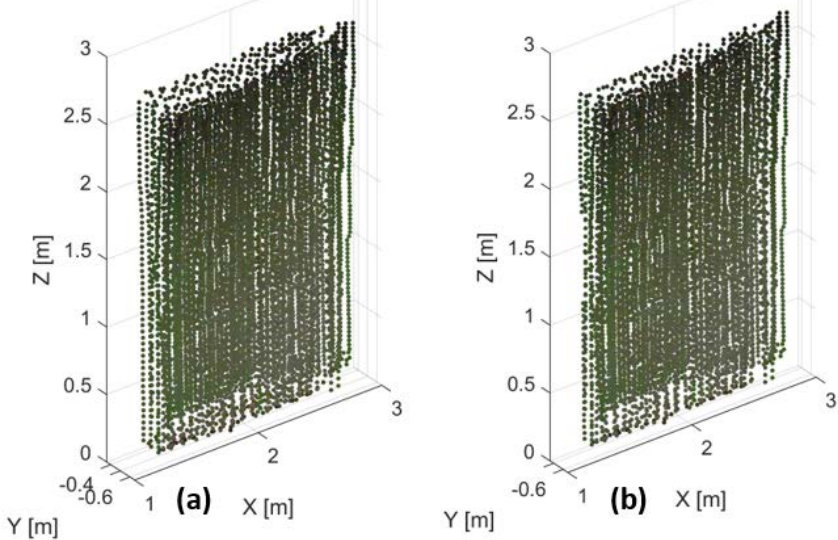

Fig. 14. Doors, (a) normal plane range, (b) estimated fine plane range.

In case of doors in Fig. 13 the algorithm was also successful. The scanning dimension is $Y$. The detection parameters are set as following, $l v l S=0.2$ and $l v l R S=1.5$.
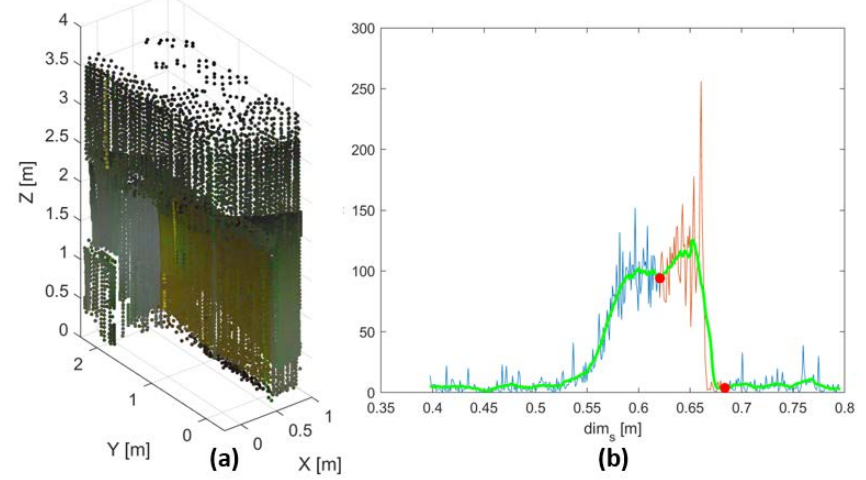

Fig. 15. Cupboard, (a) point cloud, (b) histogram of $X$ axis with marked fine range by red dots. 


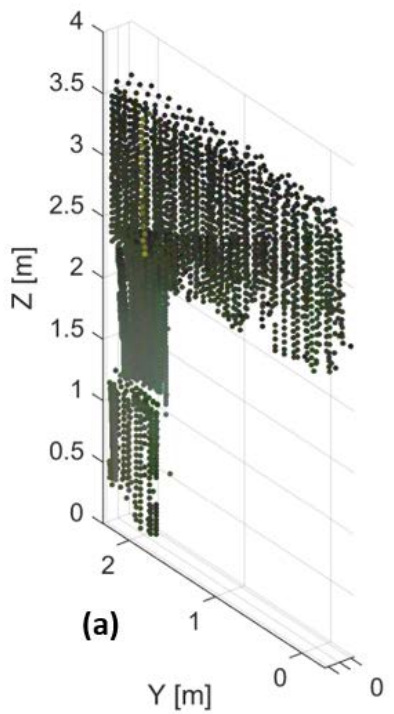

$$
X[\mathrm{~m}]
$$

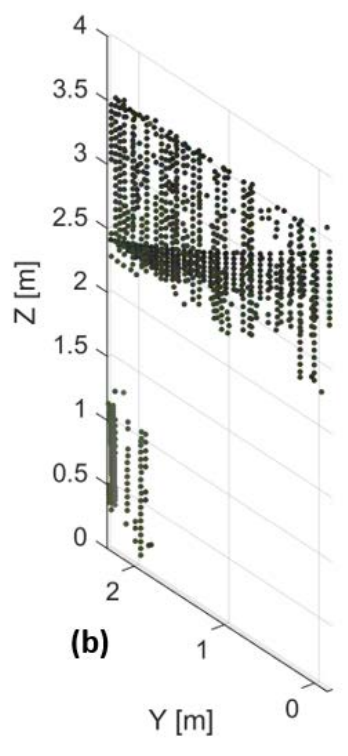

Fig. 16. Cupboard, (a) plane range, (b) estimated fine plane range.

The problematic case shows the cupboard in Fig. 15. If Fig. 16 (a) is closely analysed it is possible to notices the two close planes, which confirms also histogram in Fig. 15 (b). In practice the selected range is correct and only back wall is segmented in Fig. 16 (b). This case also showing the algorithm feature to stop in the local minimum. Always depends on the point cloud points' distribution, but if there are not any special requirements we recommend to select bigger detection range lvlS.

\section{CONCLUSION}

In this paper we presented a method for the fine plane range estimation from a point cloud. The plane range width depends on the input data quality. The point's histogram is usually noisy. We recommend its filtration before the following processing. As the practical results show, if we set the priory plane range assumption to bigger value the proposed algorithm correctly detects fine plane range. The two stage processing ensure the best elbow position estimation. The presented elbow estimation approach can be applicable to different data for its position estimation, which makes it for the general use. In the future research we will focus to overcome occurrence of local minima.

\section{REFERENCES}

[1] J. C. K. Chow, I. Detchev, K. D. Ang, K. Morin, K. Mahadevan, and N. Louie, "Robot vision: calibration of wide-angle lens cameras using collinearity condition and k-nearest neighbour regression", ISPRS International Archives of the Photogrammetry, Remote Sensing and Spatial Information Sciences, vol. XLII-1, pp. 93-99, 2018.

[2] O. A. Ramirez, A. M. Hernandez, H. R. Figueroa, M. Devy, S. P. Hernandez, and E. R. Ramirez, "A graph representation composed of geometrical components for household furniture detection by autonomous mobile robots", Applied Sciences, vol. 8, no. 11, 2018.

[3] L. Caltagirone, M. Bellone, L. Svensson, and M. Wahde, "LIDAR camera fusion for road detection using fully convolutional neural networks", Robotics and Autonomous Systems, vol. 111, pp. 125-131, 2019.

[4] E. E. Sayed, R. F. A. Kader, H. Nashaat, and M. Marei, "Plane detection in 3D point cloud using octree-balanced density downsampling and iterative adaptive plane extraction," in IET Image Processing, vol. 12, no. 9, pp. 1595-1605, 92018.

[5] X. Wang, C. Ding, Y. Wang, and X. Zhao, "A plane extraction method based on the randomized hough transform," 2017 International Conference on Computing Intelligence and Information System (CIIS), Nanjing, 2017, pp. 160-163.

[6] T. Czerniawski, B. Sankaran, M. Nahangi, C. Haas, and F. Leite, "6D DBSCAN-based segmentation of building point clouds for planar object classification," Automation in Construction, vol. 88, pp. 44-58. 2018

[7] J. S. Yoo, D. H. Kim, and G. W. Kim, "Improved LiDAR-camera calibration using marker detection based on 3d plane extraction," The Korean Institute of Electrical Engineers, Journal of Electrical Engineering \& Technology 13(6), 2018.11, pp. 2530-2544

[8] P. Chmelar, L. Beran, N. Chmelarova, and L. Rejfek, "Advanced plane properties by using level image", in 2018 28th International Conference Radioelektronika (RADIOELEKTRONIKA), 2018, pp. 1-6.

[9] P. Chmelar, L. Rejfek, L. Beran, and M. Dobrovolny, "A Point Cloud Decomposition by the 3D Level Scanning for Planes Detection," International Journal of Advanced and Applied Science, 2017, pp. $121-126$

[10] P. Chmelar, L. Rejfek, L. Beran, N. Chmelarova, and M. Dobrovolny, "Point Cloud Plane Visualization by Using Level Image," International Journal of Advanced and Applied Science, 2018, in press

[11] D. J. Ketchen Jr., and Ch. L. Shook, "The Application of Cluster Analysis in Strategic Management Research: An Analysis and Critique," Strategic Management Journal Vol. 17, No. 6 (Jun., 1996), pp. $441-458$

[12] M. Antunes, D. Gomes and R. L. Aguiar, "Knee/Elbow Estimation Based on First Derivative Threshold," 2018 IEEE Fourth International Conference on Big Data Computing Service and Applications (BigDataService), Bamberg, 2018, pp. 237-240. 